\title{
Virtual reality modeling language : et si nous assistions au dépassement de Lacan et de Vygotski?
}

Jacques Coulardeau

\section{OpenEdition}

\section{Journals}

Édition électronique

URL : http://journals.openedition.org/asp/2901

DOI : 10.4000/asp.2901

ISSN : 2108-6354

Éditeur

Groupe d'étude et de recherche en anglais de spécialité

\section{Édition imprimée}

Date de publication : 1 décembre 1998

Pagination : 357-376

ISSN : 1246-8185

\section{Référence électronique}

Jacques Coulardeau, «Virtual reality modeling language : et si nous assistions au dépassement de Lacan et de Vygotski ? », ASp [En ligne], 19-22 | 1998, mis en ligne le 16 avril 2012, consulté le 19 avril 2019. URL : http://journals.openedition.org/asp/2901 ; DOI : 10.4000/asp.2901

Ce document a été généré automatiquement le 19 avril 2019

Tous droits réservés 


\title{
Virtual reality modeling language : et si nous assistions au dépassement de Lacan et de Vygotski?
}

\author{
Jacques Coulardeau
}

1 La commercialisation des GUIs, fondées sur la psychologie de Jerome Bruner, a prouvé, au monde entier, que l'attendu central de Piaget, à savoir que les trois compétences du sujet, les compétences kinesthésique, iconique et systémique, étaient ordonnées chronologiquement dans le processus linéaire de la maturation du sujet, était faux. Ces trois compétences interviennent toujours ensemble, simultanément, même si cela se fait dans des proportions variables selon les sujets, les activités, les outils, les moments, les objectifs. La démonstration de la valeur de vérité d'une théorie psychologique est venue du succès commercial d'un produit informatique.

2 Mais depuis les GUIs, l'informatique a franchi de nombreux stades et on découvre, aujourd'hui, avec l'Internet, deux nouveaux langages, le VRML et Java, qui remettent en cause des concepts pourtant fondamentaux : à savoir le carré de Lacan, et à l'intérieur de celui-ci le concept d'Autre; et le concept de zone proche de développement de Vygotski. Le VRML et le Java ne sont encore que les premiers pas vers un monde inconnu, même si ces premiers pas sont les outils indispensables pour permettre à l'immense majorité des cybernautes d'accéder complètement à la réalité virtuelle.

3 Éliminons d'emblée le faux débat sur la virtualité de cette réalité : elle est le produit de langages-machine, de machines, d'activités humaines tout à fait concrètes; elle s'appuie sur un marché monumental; elle donne lieu à des applications industrielles et économiques sans précédent; elle met en rapport des gens de la façon la plus concrète, puisque certains cas se sont retrouvés sur le bureau de juges, dans des cours de justice et ont fait l'objet de décisions de conseils d'administration d'université. Aujourd'hui même, un chercheur comme Richard C. MacKinnon est devenu un expert du Ministère de la Justice de l'État du Texas, tout en poursuivant ses travaux au Advanced Communication Technologies Laboratory de l'Université du Texas à Austin dans le domaine des 
«Correctional Strategies for the Virtual Offender », délinquant qui n'a rien de virtuel en ce sens qu'il peut finir en prison.

There is absolutely no inevitability as long as there is a willingness to contemplate what is happening... All media are extensions of some human faculty - psychic or physical. (MacLuhan $1967: 25-26$ )

Il s'agit donc de mettre les modèles anciens à l'épreuve des pratiques nouvelles couronnées de succès commercial et de se demander les changements que ces pratiques opèrent ou révèlent dans la structuration des phénomènes couverts par ces modèles et les modifications à apporter à ces modèles. Le succès commercial doit être considéré aujourd'hui comme la « preuve du pudding ».

5 Dans un premier temps, nous aborderons le problème à partir d'une observation pédagogique d'une école internationale de langues pour ensuite seulement nous orienter vers une discussion plus large et explorer les conséquences pratiques de ces technologies que nous résumerons souvent sous les termes d'Internet et de réalité virtuelle.

\section{The Anglo World International Language School at Bournemouth, mai 1997}

6 Les écoles de langues en pays étrangers sont devenues une véritable industrie dont le marché est en train d'exploser du fait d'une demande de plus en plus grande et en développement exponentiel. En ce qui concerne l'école visitée, et donc retenue ici en matériau de réflexion, nous avons le haut de gamme garanti par le label British Council et surtout le label européen ISO 9000.

7 Elle assure l'immersion totale trois dimensions (3D), c'est-à-dire : 1 . les cours tout en anglais ; 2. l'école tout en anglais ; 3. l'environnement tout en anglais. Elle renforce, pour la majorité des étudiants, la troisième dimension par le placement en famille autochtone. La moitié de sa population estudiantine, hors-saison estivale, est faite d'étudiants qui passent une année complète à Bournemouth (9 mois). L'autre moitié, toujours hors saison estivale, y passe en moyenne trois mois. L'été, bien sûr, les proportions sont tout autres, puisque là la population estudiantine, qui double pendant trois à quatre mois, est essentiellement composée d'étudiants passant un minimum de deux semaines (obligatoires), avec une moyenne entre trois et quatre semaines. L'été, le placement est toujours, pour l'essentiel en familles d'accueil.

8 Cette école, comme la plupart, a une visée culturelle de premier niveau. Il s'agit d'immerger l'apprenant dans la culture quotidienne du pays et rien d'autre, ou presque rien d'autre, sauf pour des groupes particuliers comme les étudiants qui font une année préparatoire pour entrer dans une université anglaise. Cette école est l'une des dix ou douze institutions agréées pour cela. La culture est pour l'essentiel vue comme les us et coutumes, les habitudes de la vie quotidienne en Grande Bretagne, avec la télévision comme outil de communication culturelle quasi permanent et plutôt exclusif.

Cette approche du bain culturel est largement insuffisante. On n'atteint pas la quatrième dimension nécessaire pour que l'immersion totale soit absolument efficace. On peut penser à trois lignes principales d'action :

- L'ouverture des étudiants à la possibilité de suivre des cours spécifiques, si possible pour autochtones: technique, scientifique, culturel au sens cette fois de la culture élaborée (cinéma, littérature, arts divers, musiques...). Un couplage de cette école avec l'Université de 
Portsmouth rend des choses possibles, et l'Open University n'est pas interdite. Mais cela reste marginal, sauf pour l'année de préparation à l'entrée à l'Université qui exige un tel programme : droit, médias, histoire, business, commerce, tourisme, etc.

- Des ateliers de pratique de ces arts et de ces activités: un club théâtre existe mais fonctionne de façon aléatoire. Cependant il n'y a pas d'investissement lourd dans les cultures comme la/les musique(s), le cinéma, la/les littérature(s) ancienne(s) ou moderne(s).

- Un travail de recherche de terrain par paire ou triplette multinationale qui obligerait les étudiants à rencontrer, en plus des heures de cours, des Anglais, des autochtones, en vue de fouiller une question économique, culturelle, de loisirs, historique, ou autre. Cela entraînerait des rapports écrits et des présentations orales.

Le raisonnement derrière cette ouverture culturelle, cette quatrième dimension de l'immersion totale, est que le processus de motivation est accru et que les étudiants vont progressivement angliciser leur propre culture en l'ouvrant à l'approche anglaise ou américaine de cette culture qui est la leur. On est là dans une application directe du concept de zone proche de développement de Vygotski. Mais un second raisonnement intervient. L'anglicisation de la culture de départ de l'apprenant rend moins nécessaire le regroupement national, car l'angoisse culturelle qui découle du déracinementdépaysement-éloignement en est amoindrie. Le pays d'accueil trouve des points d'appui et des articulations sur et dans la culture d'origine. En d'autres termes, autant la culture d'origine est anglicisée, autant la culture anglaise est "nationalisée » par l'étudiant qui fonctionne ainsi. C'est donc l'intérêt de l'école de faire ce choix en ce qui concerne le succès des étudiants.

11 Mais, notre observation nous fait découvrir, dans ce domaine de la quatrième dimension culturelle, un fait qui tient de la révolution et nous ramène à l'informatique au sens large, le World Wide Web.

L'école a introduit l'informatique comme matière, marginale certes pour ne nombreux groupes, mais obligatoire, parce qu'un étudiant en Angleterre se doit d'être computerliterate. Mais ils l'ont fait avec l'objectif d'apprendre aux étudiants à se servir d'un ordinateur, sans voir l'impact énorme de ce qu'ils faisaient: utiliser un des éléments essentiels de la culture des jeunes, l'informatique, l'Internet.

13 Toutes les semaines, tous les groupes ont une session d'informatique. L'objectif principal est de remplir une tâche informatique entièrement en anglais à traiter sur l'ordinateur. Par exemple, les salaires d'une entreprise qui doit être mise en feuille de calcul, avec les retenues diverses, les impôts, etc., pour chaque employé, avec des moyennes et des paramètres multiples, avec un graphique d'un type ou d'un autre, et un résumé analytique des résultats sous la forme d'un rapport écrit. Les étudiants doivent comprendre la tâche en tant que telle, savoir faire un tableur pour la feuille de calcul, en tirer un graphique, savoir utiliser un traitement de texte pour le rapport, savoir imprimer le tout. Ils ont donc à dominer deux types d'anglais, tous les deux des anglais de spécialité, pour pouvoir remplir la tâche. Or, le fait que ce soit sur un ordinateur, le fait qu'aucun ne veut apparaitre plus idiot et illettré que son voisin, raison de plus si on ajoute quelques éléments sexuels dans ces comparaisons (du fait des cultures de certains pays), hypermotive les apprenants à comprendre l'anglais concerné et à produire le rapport demandé.

14 L'école a ensuite un Self-Access Centre. L'informatique est alors à disposition. Les étudiants peuvent faire ce qu'ils veulent, une tâche qu'ils choisissent en fonction de leur intérêt qui 
est le moteur de la motivation. Il s'agit de volontaires qui doivent se préinscrire le matin de chaque jour.

Le plus intéressant est l'utilisation actuelle de l'Internet. L'école a un site international interactif. Chaque étudiant, où qu'il soit dans le monde, entre un petit texte, illustré si cela est possible, sur un élément de culture nationale, en anglais, avec une dimension anecdotique personnelle. Une recette de cuisine, un petit événement, un incident ou accident, etc. Ce texte doit âtre reçu par d'autres. L'anglais doit en être correct. Mais il est issu de la culture nationale : fierté nationale de réussir, d'intéresser, de faire rire, à bon escient. Cela motive la qualité de l'anglais et le travail. Plus encore, l'étudiant doit comprendre et apprécier les réactions à son texte, et il doit injecter des réactions aux textes des autres, pour que son nom apparaisse le plus souvent possible. Fierté personnelle autant que nationale. On est bien là dans la quatrième dimension comme levier principal de la motivation et donc du travail de l'apprenant.

En plus, cette tentative permet de sortir l'étudiant d'un face à face entre lui, sa culture, son pays, sa nation, et le monde anglais qui l'environne. Il sort d'un face à face et entre dans une confrontation généralisée avec de nombreuses nationalités, de nombreux pays. Il devient capable de relativiser sa culture, le choc culturel avec la culture anglo-saxonne. Il s'ouvre aux autres, retrouve un équilibre, car il ne se sent plus menacé mais valorisé et enrichi.

Cette démarche, que je dois dire plutôt bricolée que réfléchie, bricolée comme une activité, sans savoir les potentialités qu'elle contient, s'appuie sur deux cultures de l'apprenant : sa culture nationale ou personnelle et sa culture informatique, et la seconde est le véritable moteur de l'opération. Et c'est là que nous entrons dans la véritable problématique de cette étude : le multimédia, la réalité virtuelle, l'Internet, le VRML et Java. Mais donnons rapidement quelques éléments sur ces deux langages nouveaux.

\section{Le VRML, Java, le langage}

Les langages de l'Internet sont le Hyper Text Markup Language (HTML), un langage spécifique pour la production et la gestion d'hypertexte, qui n'est qu'une forme particulière du Standard Generalized Markup Language (SGML). Le Java est un langage en soi qui ne traite plus du texte mais de l'image de réalité virtuelle mise en mouvement. Le Virtual Reality Modeling Language (VRML) est un langage de production d'images non animées, donc d'univers statiques. La définition de langage donnée dans le IBM Dictionary of Computing est la suivante : «A set of characters, conventions and rules that is used for conveying information » $(1994: 375)$

19 Il faut prendre en compte un autre élément à ce niveau: le language construct dont la définition donnée par IBM est la suivante :

In a programming language, a syntactically allowable program or subroutine that may be constructed in accordance with the set of rules that make up the grammar of the language. (375)

Ici apparaissent les concepts de syntaxe et de grammaire qui amplifient la métaphore du langage. On s'entend bien: il s'agit ici de langages-machine. N'importe qui peut construire des macro-opérations qui ont le statut de language constructs, formules de calcul ou opérations et expressions fréquentes, que l'on met en mémoire avec des procédures raccourcies d'appel, ce qui permet de gagner du temps. Le VRML produit un 
univers virtuel 3D et le Java une animation, des mouvements dans ces univers construits. Ils intègrent donc des calculs et des éléments graphiques.

\section{Le système-auteur}

C'est l'invention d'une procédure complexe de traitement de données, texte ou objets. Il s'agit de remplir les cases vides avec des paramètres quelconques pour que ce systèmeauteur fonctionne. Un carnet d'adresses électroniques est un tel système-auteur. Certains de ces systèmes auteurs sont très complexes mais les techniques du Plug-and-Play en produisent certains d'une facilité d'utilisation défiant l'entendement. Dans certains domaines on entend par système-auteur des logiciels dédiés, c'est-à-dire dont l'objectif est clairement défini : exercices structuraux de grammaire par exemple. Ces systèmes auteurs préétablis peuvent être customisés. D'où les concepts de ready-to-use et userfriendly.

\section{Le VRML}

Fondamentalement c'est un tel système-auteur. Il contient des formes, des couleurs, des motifs, des positionnements dans l'espace sur trois axes ( $\mathrm{x}, \mathrm{y}$ et $\mathrm{z}$ ), du texte, des textures, des matériaux au sens de pierre, ivoire, métal, etc., des grains et autres éléments d'aspect. Chacun de ces éléments est ouvert quant à ses paramètres. Ensuite nous avons des outils pour choisir les éléments et fixer les paramètres, ou imposer des métaformes aux formes choisies, métaformes qui ne sont que des outils préfabriqués à partir de la possibilité dans un logiciel de dessin. Le VRML n'est qu'un outil de dessin ou de sculpture. On peut inclure dans ces mondes imaginaires des éléments réels en motifs ou objets pour ensuite pouvoir être animés. Dans une interview, Cindy Reed, directrice technique de Yglesias Wallock Divekar, Inc., déclare : « impossibilité de se prémunir contre la copie illicite de modèles », ce qui explique que son entreprise ne met pas sur le marché libre ses propres produits en VRML. Signalons les « hackers » qui volent et les «nukes » qui détruisent. Mais aussi les techniques de verrouillage et les «antinukes». Toute personne possédant les langages VRML et Java peut injecter, s'approprier ou détruire ce qu'il veut dans n'importe quel monde qu'il pénètre.

\section{Java}

Ce logiciel ne représente rien de vraiment différent du niveau précédent, si ce n'est qu'il ne gère plus des formes, mais des mouvements de ces formes. Mouvements abstraits (rotation, rebond) paramétrés (force initiale, vitesse, sens, direction, obstacles, élasticité). Mouvements généraux : course, marche, saut, plongeon. Mouvements décomposés en fonction du corps ou de la machine. On retrouve ici la Chorésynthèse d'Edmond Linval. L'auteur peut toujours changer ce qu'il veut dans son univers sans obligation de tenir compte du réalisme, ou du vraisemblable.

\section{L'interactif}

Rencontre, à un moment $\mathrm{X}$, de plusieurs personnages créés par plusieurs utilisateurs, purement aléatoire dans cet univers. On a alors de vrais jeux de rôles, avec ou sans règles du jeu. Ces règles, si elles existent, peuvent être intégrées dans le programme comme des limites, mais aussi peuvent exister comme de simples codes éthiques. On peut avoir un maître du jeu ou aucun. Tout est possible. Le propriétaire du site peut rétablir le paysage à la session suivante. Ce concept de propriétaire du site est fondamental. Notons que cela pose des questions juridiques au niveau des comportements répréhensibles et qui ne peuvent être réprimandés ou réprimés que dans le cadre des règles, des us et coutumes du site concerné. Notons la dimension économique de cette révolution : 
Ce public gigantesque commence à attirer une force qui modifiera à jamais le Web : le commerce. [...] L'édition sur le Web, le marketing sur le Web, la publicité sur le Web et le développement de technologies pour le Web sont des industries pesant des milliards de dollars. (Stanek 1996 : 738-739) possibilité pour tout un chacun de créer son site et de devenir maître du cyberspace. Il s'agit d'une industrie et d'un commerce monumentaux qui se fondent sur une langue industrielle. La possession de cette langue industrielle est un objectif majeur de qui veut dominer dans le domaine, et là une autre industrie apparaît qui est celle de la pédagogie de cette langue, cette fois en langue naturelle, mais une langue de spécialité.

\section{VRML et Vygotski}

L'Internet est une mine inépuisable mais dans le plus parfait désordre. Il est interactif par principe et permet l'hypernavigation. Aujourd'hui le commerce s'y installe et cela donne à l'Internet un rôle et une force qu'aucun réseau de diffusion de l'information n'a jamais eu. Certains mettent en parallèle la télévision et l'Internet (ou/et la galerie marchande), d'où la nouvelle trinité du monde de la communication moderne, une trinité qui est formée de quatre éléments, car il ne faut surtout pas oublier l'autoroute qui est la matrice mentale de cette circulation de masse et en réseau.

The logic is dialectical: the greater the dependence of the individual on television, the less dependent $\mathrm{s} / \mathrm{he}$ becomes on the public sphere which is being displaced in practice ; and the more such a public sphere, particularly in its architectural/ compositional aspects, withers away. Of course, what television cannot supply in the face of the withering away of the public sphere is its quality as an interactive context. This is where the dialectic emerges. When television and other forms of broadcast have all but saturated global culture and the media corporations have exhausted the franchising of their recycled family serials, the Internet appears just in time to save us from the tyranny of broadcast that is said to have subjugated us for so long. (Holmes 1997 : 34)

La force de l'Internet est identifiée comme la possibilité d'associer les caractéristiques suivantes :

the socio-spatial as well as the ideological preconditions of virtual communities, connectivity and reciprocity, information storage, interactivity, broadcast, extending the properties of speech, writing and the image. (Holmes 1997 : 35-36).

C'est lors de la première conférence du World Wide Web en 1994 que Tim Berners-Lee et Dave Raggett ont convoqué un atelier pour discuter de la réalité virtuelle et de son utilisation sur le Web. C'est là qu'est né le VRML. L'image devient donc un lieu de production.

Vygotski pose que l'apprenant, et même plus généralement le sujet, ne peut s'intéresser à ni acquérir un savoir que si ce savoir fait partie de la «zone proche de développement ». C'est quand on connaît déjà quelque chose, quand on comprend déjà quelque chose, que l'on peut s'ouvrir à autre chose qui est proche de cette connaissance et de cette compréhension, et c'est la visée d'acquisition de ces nouvelles connaissances et compréhensions qui entraîne et motive le développement.

L'apprentissage n'est valable que s'il devance le développement. Il suscite alors, fait naître toute une série de fonctions qui se trouvent au stade de la maturation, qui sont dans la zone proche de développement. (Vygotski 1985 : 275.) 
On voit ainsi que la conception de Vygotski pose le rôle du maître comme devant déterminer les objectifs de l'apprentissage pour ainsi utiliser la zone proche de développement et provoquer la maturation, ou du moins la réaliser et l'accélérer. Un tel maître devant l'Internet n'a plus le pouvoir de déterminer quoi que ce soit. Les jeunes pénètrent dans cet univers et hypernaviguent sans fin. Ils éprouvent un sentiment d'extrême excitation face à ce voyage suprême qui les emmène dans des domaines qui n'ont, pour certains, aucun lien avec leur zone proche de développement. Ce sentiment de jouissance suprême vient de trois éléments. D'une part, la dimension ludique. Ensuite, la domination de la machine, dimension de la puissance phallique. Enfin, c'est l'immersion totale dans des mondes inconnus et a priori fascinants, la dimension pulsionnelle.

31 Les jeunes ne s'y noient jamais, puisqu'il suffit de rebooter d'un simple mouvement de doigt pour que la mort assurée soit niée, mort en définitive virtuelle, d'autre que le sujet. Quoi d'ailleurs? Mais c'est là que le VRML et le Java interviennent pour démultiplier les capacités du média. Richard C. MacKinnon (in Jones 1997) discute longuement, sur la base des réflexions de Michel Foucault (1979) et pose une problématique fondamentale. La person se divise en deux, le body et le self (nous ne tenons pas à traduire ces termes). Le self peut avoir ou non une dimension spirituelle allant jusqu'à l'âme, ou en rester à une conception psychologique de simple sujet, pensant, éprouvant... Or ce n'est jamais cette person qui entre dans le monde virtuel. C'est une persona, pure invention de la person qui projette un personnage dans le monde virtuel, personnage qui le représente électroniquement car il est l'outil de son action, mais aussi, qui peut être le résultat d'un transfert positif ou négatif d'autres modèles et pas seulement de ses propres pulsions, même si celles-ci interviennent toujours d'une façon ou d'une autre dans cette persona et son comportement.

Figure 1. L'interaction dans un univers virtuel

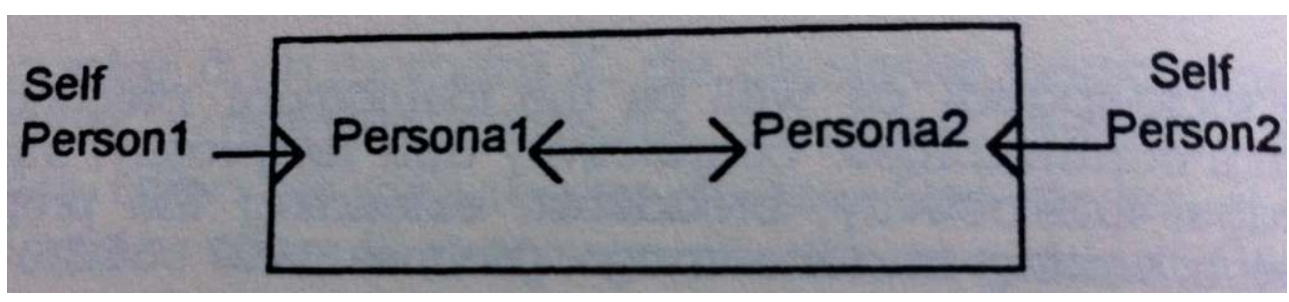

Ainsi, trois conséquences en découlent. D'une part, la distance entre la person et la persona protège complètement la person. Un auteur n'est jamais en rien son personnage. D'autre part, ce n'est que la persona qui meurt éventuellement dans le monde virtuel. L'échec n'est jamais celui de la person qui peut toujours recommencer et réussir éventuellement. Projection de soi-même ou d'une image de soi-même dans la persona, donc une certaine identification, mais partielle et totalement contrebalancée par une distanciation, une virtualisation de la relation et verrouillage de la distanciation dans l'anonymat. Mais plus encore, la réalité virtuelle permet à la person, grâce à cette persona, d'échapper au temps et à l'espace. « VR allows us to overcome the limits of being in one location at one time. » (Holmes, 1997 : 39).

Danger de la totale coupure du monde? Non. Protection, car le retour est toujours et facilement possible. Aventure, car le sujet entre dans ce monde infini avec toute sa charge personnelle, sa culture, ses sentiments, ses pulsions, ses envies, ses savoirs, etc. Expérience totale. Il va être confronté à des savoirs complètement inattendus, et le jeu 
voudra qu'il y entre, y pénétre et en revienne avec quelque chose, un souvenir, un savoir. Giovannisme à trois niveaux : le ludique, le phallique et le pulsionnel. Mettre des apprenants sur l'Internet, c'est nier la logique de Vygotski et de sa zone proche de développement.

\section{VRML et Lacan}

Le carré de Lacan se trouve déstructuré par l'impact de l'Internet et de la réalité virtuelle. Le Moi apparaît très fort dans le ludique. C'est un des moteurs essentiels de la pénétration de ces mondes virtuels, s'appuyant sur un langage personnel, brut, qu'on peut injecter dans le réseau, langage égocentrique évident. Jones est plein de tels exemples :

No longer do we, as members of the group, belong to the community, rather the community belongs to us... We are struck, as we use the Internet, by the sense that there are others out there like us... (1997: 16)

Nous pourrions considérer la présence de I dans les citations du Web de l'article de Joseph Schmitz «Structural Relations, Electronic Media, and social change : the Public Electronic Network and the Homeless » (in Jones, 1997), présence massive et dominante. Et nous pourrions pousser un peu plus loin cette étude de l'auto-expression égocentrique dans ce discours électronique pour voir apparaitre deux antagons. L'antagon auquel le je se dit appartenir, et l'antagon auquel cette communauté, et donc ce je, est opposée.

Cela nous amène au deuxième concept, l'Idéal du Moi. C'est d'abord la persona que le sujet égocentrique met en place sur le réseau, fausse, maquillée ou déguisée. Puis c'est le désir d'appartenir à une communauté, à un groupe qui représente comme une perspective pour l'individu. Partout l'Idéal du Moi est présent, et toujours dans le cadre d'une définition communautaire, même si le terme de communauté n'a pas la valeur sociologique traditionnelle. Ainsi, David Holmes souligne dans son article :

With the Internet we are witnessing, on the one hand, the extraordinary reach of interactive networks and, on the other, a shrinking of geo-spatial worlds to computer screen culture... But the degree of bodily isolation and solipcism varies between technologies. The walkman seals off hearing, the VR helmet seals off vision and sound, and prototypes of cybernetic clothing aim at the ultimate VR case of sealing off all the senses. (1997: 38-39)

On semble ici prendre le contre pied de MacLuhan pour qui une invention technique est l'extension d'un organe des sens :

The wheel is an extension of the foot. The book is an extension of the eye... Clothing, an extension of the skin... Electric circuitry, an extension of the central nervous system. Media, by altering the environment, evoke in us unique ratios of sense perceptions. The extension of any one sense alters the way we think and act -the way we perceive the world. When these ratios change, men change. (1967: 31-41)

On voit bien l'enjeu de ce débat. Tout comme certains nient la réalité communautaire des communautés virtuelles (virtual communities, le terme est de Howard Rheingold), certains ne voient que l'enfermement par rapport à l'environnement immédiat sans voir l'ouverture énorme que cet enfermement représente sur un monde culturel immense, sur une vie communautaire nouvelle, d'un type nouveau, fondée pour l'essentiel sur l'interaction. 

dans ses voyages. De plus l'apprenant découvre des savoirs, ou simplement des données, que le maître n'a aucune chance de connaître. L'apprenant ne l'est plus par la décision du maitre, ni par son guidage, ni par son savoir. L'apprenant est entièrement libre et responsable de son acquisition de savoir sur l'Internet. D'autant plus qu'il peut accéder à l'Internet chez lui, en dehors du milieu scolaire. La pénurie des outils de transmission ou de stockage du savoir est depuis longtemps terminée, et cela va aller croissant dans les années qui viennent. De plus en plus d'apprenants vont avoir accès à l'Internet.

Il y a donc un décentrage complet du sujet de Lacan par la marginalisation de l'Autorité de l'Autre. Et nous parlons bien sûr d'Autorité, pas de pouvoir. Le sujet se recentre entièrement sur lui-même, sur son Moi et son Idéal du Moi, avec en arrière l'autre des 
pulsions et des désirs. En face de lui une immense myriade d'Autres sans Autorité et sur lesquels le sujet peut exercer presque librement son propre pouvoir, sa propre Autorité. Il peut nier l'Autre, le rejeter, l'asservir, toujours par sa persona interposée. Du moins tenter sans aucun risque pour sa person. Le sujet peut alors franchir toutes les limites, à condition de contrôler le langage nécessaire, langage-machine et langue naturelle, pour l'essentiel de l'anglais. On peut à la limite voir cette persona comme une incarnation de l'Idéal du Moi, mais sans une Autorité castrante ou frustrante derrière, investi de sa propre Autorité sans limites. On retrouve ici un débat que Harris Breslow tente de résumer (in Jones, 1997). Sa remarque: "Alienation has two dimensions: one can be alienated from oneself as well [as] from others. » $(1997: 253)$ fausse le débat.

La première aliénation est celle de l'homme exploité par la machine, par l'économique de la machine. Cette aliénation ou exploitation de l'homme s'est affinée et renforcée.

La seconde aliénation n'est en rien nouvelle. On a accusé bien des médias de provoquer cette aliénation, c'est-à-dire de causer le retrait de l'individu en lui-même, et donc son retrait de la compagnie des autres. Le livre, la télévision, un walkman. Aujourd'hui le sujet a pour objectif premier de sans cesse élargir son savoir, enrichir sa culture, et échanger, par tous les moyens à sa disposition, l'information, la réflexion et l'imagination qui lui sont propres.

\section{Les avantages du VRML}

L'utilisateur de l'Internet est self-centered, self-initiated, self-motivated et self-interested. Le Moi et l'Idéal du Moi sont devenus le centre du sujet. Le sujet a l'initiative totale de sa propre navigation informatique. Sa motivation ne peut venir que de l'intérieur de luimême. Il recherche d'abord et avant tout la satisfaction de ses intérêts vitaux du moment. Cela transforme complètement la société, mais plus encore, le processus didactique et pédagogique.

49 La pédagogie est autocentrée sur l'apprenant qui se déclare le centre du processus et exige donc d'être ce centre. La montée de cette exigence est très nette, même si parfois elle est contradictoire, avec ceux qui tirent vers le haut et ceux qui tirent vers le bas. Cela implique que nous prenions en considération totalement les cinq stratégies cognitives (visuel, langue orale, langue écrite, tactile et culturel) de l'apprenant et ses intérêts. C'est d'ailleurs pour cela que le multimédia et l'Internet sont si fascinants pour l'apprenant, car l'apprenant est dominant visuel.

50 La pédagogie est auto-initiée par l'apprenant. C'est lui qui démarre la machine. Il doit donc être à l'initiative des choix et des sujets. On doit aujourd'hui de plus en plus faire sentir à l'apprenant le besoin de savoir, et satisfaire son besoin. L'Internet devient un outil important car c'est bien l'apprenant qui a l'initiative de l'hypernavigation.

51 La pédagogie est auto-motivée par l'apprenant. Tout doit venir de lui, sinon il ne suivra pas la voie nécessaire pour atteindre le savoir qu'il recherche. Il doit être convaincu que c'est lui qui a déterminé ce savoir pour garder totalement l'initiative maximum et surtout rester sur la route qui mène au savoir visé. Sinon il se démotive et il va partir dans des chemins de traverse. L'hypernavigation permet cela. Ce n'est plus une cassette de laboratoire de langues ou une disquette d'exercices structuraux sur un vieil ordinateur 286. 
La pédagogie est auto-intéressée par l'apprenant. Il n'acceptera de prendre en considération que ce qui l'intéresse, que ce dont il est convaincu que cela l'enrichira, enrichira son plaisir immédiat (ludique à court terme) ou enrichira son Autorité ultérieure dans la société (phallique à plus ou moins long terme). Le maitre doit donc passer beaucoup de temps à convaincre l'apprenant de l'intérêt de ce qu'il lui propose, ou plutôt de l'intérêt de ce que l'apprenant suggère. C'est cet intérêt qui amènera l'apprenant à écarter certaines choses et à en rechercher d'autres. Cela est un bon gardefou dans l'hypernavigation.

Figure 3. Topographie ou architecture de l'établissement scolaire dans sa centration sur le Internet Multimedia Self Access Center within Guided Autonomy

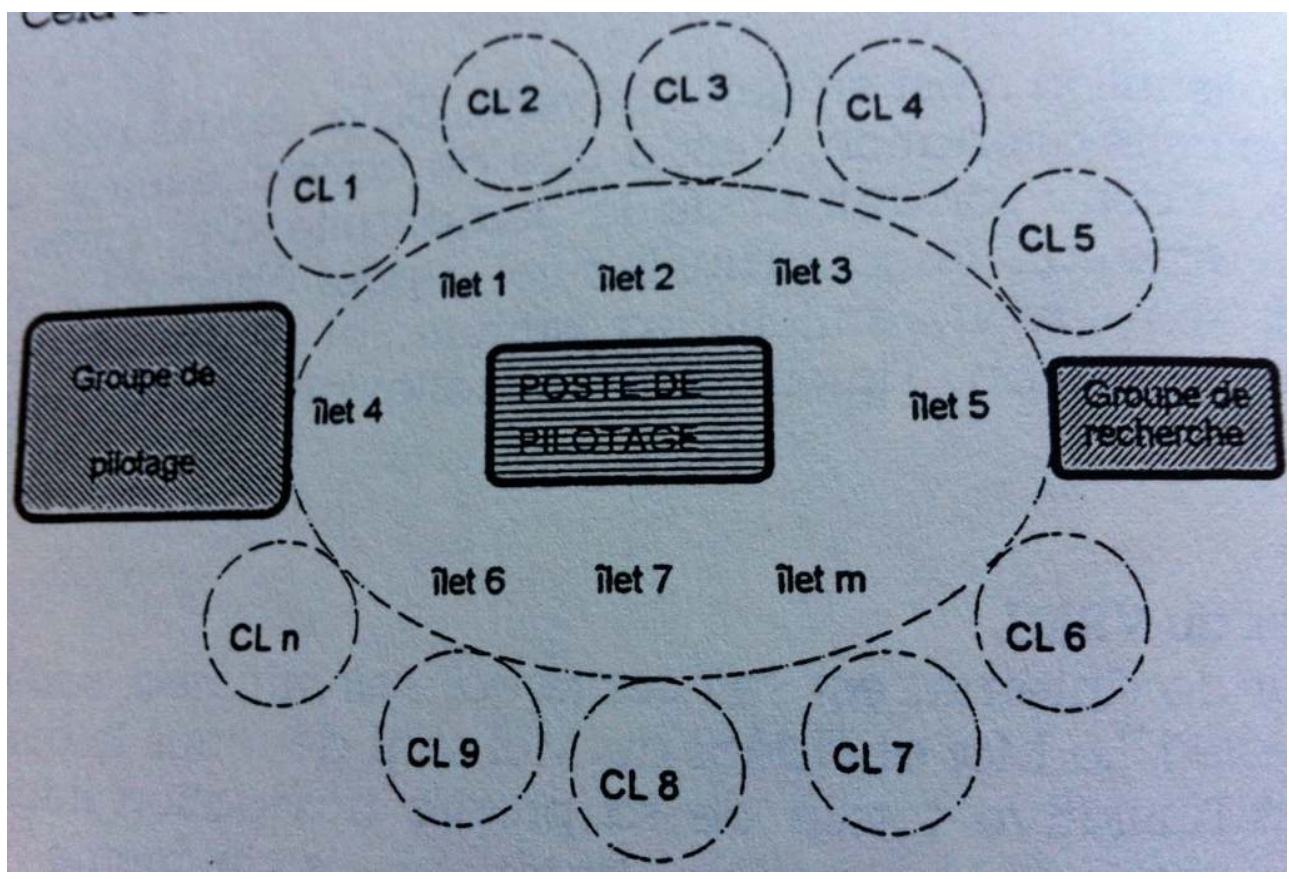

Notre pédagogie doit donc se fonder sur la coopération entre les apprenants tout autant qu'utiliser leur autonomie maximum. La classe ne doit plus être une forme obligée de transmission du savoir. La classe doit exploser comme forme privilégiée de travail. L'enseignant doit inciter la classe (le groupe), ou des sous-groupes dans la classe, à chercher le savoir où il se trouve. L'éducation doit se centrer sur un savoir aujourd'hui explosé et se recentrer sur des centres de ressources. L'enseignant doit inciter le groupe à se choisir une perspective, et à déterminer la procédure pour l'atteindre. Il ne doit pas enfermer l'apprenant dans l'Internet. Le maître doit rester plurimédia plus que multimédia. L'apprenant, seul ou en petit groupe, doit être convaincu qu'il travaille pour lui-même et que son travail est une recherche de savoir entièrement centrée sur luimême, sur son intérêt. Le maitre devient un guide qui conseille parfois et qui résout les blocages quand il y en a. Quand un certain niveau de savoir est atteint, la classe peut se reconstituer, et cela est essentiel, pour discuter, confronter, présenter, enrichir, ce que chacun a trouvé. La mise en commun, le partage du savoir, est fondamentale. Il s'agit pour chacun d'apprendre à communiquer le savoir découvert aux autres, à écouter les autres et ensuite à discuter des contradictions et des différences. Ce processus de confrontation doit être fondé sur le dialogue, la parole, le langage et doit viser à une construction commune qui n'exclut pas les différences mais les pose comme nécessaires à 
la suite. De nouveaux objectifs apparaissent et le maître doit veiller à ce que chacun soit engagé dans le processus, et que chacun, avec ses moyens, puisse participer au processus. Le travail devient une spirale, une came, comme dirait Culioli. Le maitre n'est plus qu'un homme-ressources méthodologiques. Il indique où le savoir peut se trouver. Il suit et aide les efforts des apprenants dans leur recherche. Il fournit les outils d'analyse et de synthèse nécessaire à la construction d'un savoir à partir de données brutes. Il est l'arbitre de la confrontation, celui qui veille à ce que le but fixé soit atteint. Et la machine n'est que l'outil qui va permettre le partage de ce ou ces savoirs avec des groupes éloignés, avec le monde entier. Le savoir commun ne peut être qu'un consensus contradictoire. To agree to disagree. Ce qui implique que je comprends pourquoi l'autre a un point de vue différent du mien, et pourquoi j'ai le point de vue que j'ai.

\section{Les dangers du VRML}

Nous passerons rapidement sur le problème de la criminalité qui utilise l'ordinateur et l'Internet comme outil ou média du crime. Un cas fait école aux USA : il s'agit de Kevin Mitnick (in Jones 1997). Cet homme utilisait l'ordinateur pour s'approprier des données qu'il revendait éventuellement, et surtout pour faire des malversations financières, par l'intermédiaire de cartes de crédit par exemple. Un livre important est The Poet de Michael Connelly (1996). Ce livre met en scène un criminel qui occupe un poste extrêmement élevé dans la hiérarchie du FBI, et qui utilise cette position et les réseaux informatiques qui l'accompagne pour organiser une série d'assassinats de policiers, réalisant ainsi sa vengeance face à son père, policier qui avait abusé de lui pendant des années quand il était enfant. On note ici la dimension sexuelle systématiquement donnée à ces crimes, avec un faux suspect qui fait dans le commerce des images pédophiliques par l'intermédiaire du Web. C'est une sorte de mode dans la littérature américaine actuelle, qui correspond à une vision sexualisée de ces machines, révélant désir et peur dans le public. Notons que les X Files utilisent aussi cette dimension.

Le deuxième danger, ce sont les crimes commis à l'intérieur même d'un monde virtuel. Il faut d'abord que ce monde virtuel pose ce comportement comme criminel. Il faut ensuite prouver que la person qui est derrière la persona pratique les mêmes actions dans le monde réel. Ce ne sont des délits relevant des tribunaux qu'à ce moment-là. Les USA ont tenté de réguler ce problème avec le Communications Decency Act (février 1996), mais la Cour Suprême l'a rejeté comme contraire à la Constitution, particulièrement au Premier Amendement.

Nous allons par contre développer les dangers pédagogiques et psychologiques.

Le premier est la perte de contact avec le monde réel et l'enfermement complet dans un monde fictif ou virtuel. On parle d'enfermement psychotique. Notons tout de suite que cela n'est pas typique uniquement de l'Internet. On parle dans les mêmes termes de la lecture obsessionnelle de certains individus, que ce soit de la littérature de haut niveau, ou que ce soit de la littérature populaire. Même approche face à la télévision accusée de tous les maux du monde quand elle devient obsessionnelle, ou encore les jeux électroniques et les jeux de rôles. En fait, il s'agit de la part de la société de critiquer et condamner, avec les mots à la mode au moment de cette critique et de cette condamnation, le fait qu'un sujet puisse trouver son énergie vitale en dehors de cette même société. Cela est d'autant plus fort aujourd'hui du fait d'une évolution qui a commencé il y a déjà deux siècles (les révolutions américaine et française et leurs 
déclarations des droits de l'homme et du citoyen, puis la révolution industrielle), une évolution qui progressivement libère l'individu à tous les niveaux de son être de la dépendance qui était la marque première des sociétés féodales ou anciennes. C'est l'émergence de la société civile. (cf. Breslow 1997: 248-249). La société civile d'aujourd'hui, ou plutôt la société politique d'aujourd'hui, dans nos sociétés avancées et de liberté individuelle, essaie d'envahir la sphère privée et de la régenter. En fait, cette longue évolution de l'émergence de l'individu comme seule source et énergie de sa liberté est en train enfin d'arriver à échéance. Les moyens d'information et de culture, les outils de la liberté suprême, à savoir la liberté intellectuelle et mentale sont à portée de la main. Les moyens nouveaux permettent de développer l'imaginaire des individus, des gens. $\mathrm{Ne}$ serait-ce pas là le danger réel ?

Le deuxième est la noyade totale dans un flux désordonné de données. Cela va créer des névroses méthodologiques. Cela va causer la perte ou le non-développement de l'esprit d'analyse, de l'esprit de synthèse, de l'induction et de la déduction. On va se mettre à penser de façon purement syncrétique, impressionniste. Les données les plus belles, les plus frappantes, les plus aguichantes seront les meilleures. Pire encore. Certains vont, en toute naïveté et innocence, appliquer un certain pouvoir d'analyse, un certain pouvoir de synthèse, une certaine induction et une certaine déduction à des données tellement informes et farfelues qu'ils ne pourront en arriver qu'à des conclusions erronées, des interlangues informationnelles, c'est-à-dire d'abord et avant tout informes. Il va y avoir régression, perte sèche de pouvoir cérébral. L'humanité tout entière va en revenir à un charabia primitif. De tels arguments sont la marque d'esprit frileux. L'encyclopédie de Diderot, la Bible entre les mains de tout le monde ont subies les mêmes critiques. De plus, tout cela est un peu dépassé, car d'ores et déjà, pour le marché des chercheurs, des CDRom d'analyse et de mise en ordre et en forme des données brutes recueillies à droite et à gauche, sont disponibles. Ces produits se multiplient. Le danger alors va être que ces produits imposent une méthodologie, une méthode, et donc un point de vue dont les conclusions seront dépendantes. Mais il y a aussi des produits qui donnent divers points de vue, y compris des points de vue contradictoires, alternatifs, marginaux, voire contestataires. Les données sont alors mises en forme selon les choix du sujet et celui-ci peut pousser son analyse avec des paramètres variables et modifiables par l'utilisateur. On voit alors que le flot ou le flux de données va demander un travail méthodologique préalable pour pouvoir le passer au crible de tels analyseurs de données, et donc pour savoir choisir les analyseurs pertinents pour les données concernées et les objectifs visés. Notre enseignement va pouvoir devenir méthodologique plus qu'informationnel. Ainsi deux pouvoirs phalliques vont s'affronter dans le cybernaute, le pouvoir des simples données que l'on épelle en chapelets, ou le pouvoir de la mise en forme des données et de la présentation d'un savoir et discours construits relatifs et conscients de leur relativité.

Certains vont encore plus loin en prétendant que l'utilisateur de la réalité virtuelle perd tout sens du temps et de l'espace réels et se trouve enfermé dans un temps-machine, un espace-machine, une société-machine coupés de la réalité. C'est oublier qu'entre person et persona il y a une distance et que justement cette distance est constitutive et constructive de l'Idéal du Moi et la base même de la jouissance du Moi.

On voit donc que pédagogiquement, la réalité virtuelle transforme l'apprenant, le maître et les rapports entre eux. C'est souvent parce que l'on est contre cette transformation qui nie le pouvoir (pouvoir qui cache l'absence d'autorité) du maître, que l'on se déclare contre la réalité virtuelle. 
61 Le danger suprême est souvent cité comme étant parfaitement illustré dans deux films dont Stephen King a renié toute paternité, à savoir Lawnmower Man I \& II. Ces films ont eu un impact sur le public probablement énorme, si on en juge par les masses de spectateurs et d'acheteurs des cassettes.

62 Le premier film pose un handicapé mental, sous la garde d'un prêtre qui le fouette régulièrement quand il ne remplit pas correctement ses devoirs de nettoyage de l'église. Il est utilisé par un jardinier professionnel pour tondre les pelouses des maisons riches. Son voisin, un chercheur en réalité virtuelle travaillant sur un projet totalement top secret de développement des capacités mentales par l'association de la réalité virtuelle et d'une drogue X non spécifiée, décide de passer des singes à ce cobaye humain. Notons l'horreur d'utiliser un handicapé mental. Le traitement est efficace et le jeune homme se met à assimiler des masses de savoir et de processus mentaux de plus en plus complexes. Il en est transformé, se libère de son prêtre tortionnaire, libère son corps de ses carcans vestimentaires et sexuels. Au détour d'une manipulation par le chef de service qui change la drogue $X$ pour une drogue beaucoup plus puissante, il devient un autre homme et ne rêve plus que de pénétrer totalement dans le monde virtuel avec lequel il joue. Il y arrive et perd toute matérialité corporelle pour n'être plus qu'une persona n'existant que sur les réseaux de communications. Le deuxième film va beaucoup plus loin car le héros retrouve une matérialité corporelle et obtient des pouvoirs de plus en plus puissants. Les expériences secrètes se poursuivent. Il devient une arme particulièrement dangereuse : il est capable de décomposer la matérialité corporelle d'hommes réels. Mais il pénètre dans un monde de réalité virtuelle nouvelle et se transforme en gourou mondial qui s'adresse à l'humanité tout entière et peu à peu essaie de la faire pénétrer dans ce monde virtuel où elle deviendrait parfaite mais immatérielle. On retrouve là un discours fort contre les sectes spiritualistes suicidaires. Heureusement le tout échoue grâce au retour vengeur du chercheur initial qui avait été écarté.

Les dangers réels que l'on rencontre dans ce film ont peu à voir avec la réalité virtuelle. Il s'agit des discours sectaires spiritualistes actuels. Il s'agit de recherches militaires ou paramilitaires qui se font dans certains laboratoires secrets, etc. En fait on dénonce une société fasciste qui essaie d'utiliser le spiritualisme de certaines sectes intégristes investi dans la modernité de la réalité virtuelle. Cela bien sûr active les discours anti-modernistes et passéistes, les discours moralistes aussi. Bref cela semble encourager le développement dans certaines sections de la société d'un ordre moral. On oublie qu'une oeuvre imaginaire, aussi idéologique soit-elle, n'est qu'une oeuvre imaginaire et doit être traitée comme telle et donc soumise à une critique artistique d'abord, idéologique ensuite. Mais toute critique sous-entend une grille de lecture et certains oublient de nous donner leur grille de lecture et jouent sur la peur séculaire d'un au-delà toujours vu somme infernal. Mais il est sûr qu'une oeuvre de l'imagination ne saurait avoir de valeur scientifique ou objective.

64 L'individu utilisateur trouve toujours le monde qui va lui permettre de réaliser, virtuellement, ses pulsions, ses besoins, ses désirs les plus secrets, et produire des pervers. Mais ce monde ne fera pas d'un être X un être Y. Ce monde n'a aucun pouvoir génétique et a peu de pouvoir psychologique. Ce monde n'a un effet sur une person que dans la mesure où il contient des éléments qui sont en germes plus ou moins réalisés dans cette person. La persona devient alors l'intermédiaire de ces pulsions et de ces désirs. Cela pourra rester secret, si la person ne révèle pas son identité. Cela pourra devenir public dans le cas contraire. 

de mondes inconnus peut devenir une psychologie de conquête. On réinvente la colonisation. Le problème posé est donc la capacité d'un site à se défendre contre de telles attaques, de tels manquements à l'éthique du site. Ici nous avons le syndrome Mr Bungle dont la persona a, sur un site quelconque dont l'éthique semble exclure un tel acte, violé la persona d'une autre personne. Le site a mis à mort la persona de ce Mr Bungle, qui s'est alors doté d'une nouvelle persona plus respectueuse des règles, et qui a finalement quitté le site. La peine était disproportionnée par rapport au crime, mais il n'en reste pas moins que l'on a bien là une psychologie de cybernaute qui dérive vers un extrême colonisateur, de pouvoir excessif et devenant par là même criminel en ce sens qu'il ne respecte plus les règles éthiques ou autres du site. Cela ne prouve en rien que la psychologie de la person qui est derrière ce Mr Bungle soit celle d'un être violent et dominateur. Il n'a peut-être, et probablement, que projeté des pulsions contradictoires à ou de son être réel (in Jones 1997 : 213)

La psychologie de découverte de mondes inconnus, si la person en a le pouvoir informatique, peut produire une persona qui, de l'acquisition de savoir nouveau, peut dériver vers l'appropriation du monde virtuel, voire même des biens des persons qui sont derrière les personae qu'elle rencontre dans ce monde. On a là le syndrome Kevin Mitnick dont nous avons déjà parlé. C'est la psychologie du hacker, du pirate, du pilleur. Là encore le problème de la défense des mondes virtuels contre de telles persons est complexe et difficile, car une telle person utilise le monde virtuel pour s'attaquer aux biens et aux possessions d'une autre person. Cela relève alors franchement de la loi réelle et de la justice du monde extérieur, car il y a simplement vol ou spoliation de biens. (in Jones 1997 : 212)

67 Ces deux attitudes sont des extrêmes, mais ce n'est pas la réalité virtuelle qui crée le délit ou le crime. Elle n'est plus qu'un lieu de réalisation virtuelle du délit dans les deux premiers cas, ou un outil de réalisation réelle du crime dans le deuxième cas. Mais accuser un artiste, même mauvais, de jouer à l'exhibitionniste pour flatter le voyeur dans le public pour vendre sa marchandise est un jugement moral, et le passage à la loi serait fort difficile. Pour trouver un tel procès en France il nous faut remonter à il y a longtemps (Baudelaire et Les Fleurs du Mal, Flaubert et Madame Bovary, au siècle dernier, et bien sûr le film La Religieuse d'après Diderot ou la pièce La Paix d'Aristophane au début des années 1960.

\section{VRML et Extension de l'Homme}

Nous en arrivons alors à la question fondamentale. Mais de quoi la réalité virtuelle estelle l'extension?

1. La réalité virtuelle est un réseau. À ce titre, elle est l'extension du système nerveux central et périphérique pour reprendre les termes de MacLuhan (1967). Elle permet ainsi l'hypernavigation qui remonte par les sens et le système nerveux périphérique au système nerveux central. On pourrait être plus fin et dire que le casque est l'extension de la vision, le gant l'extension du toucher digital, la combinaison l'extension du toucher corporel total. En tant que réseau, elle est aussi une extension des relations et connexions sociales, car elle permet de dépasser et poursuivre ou amplifier les relations sociales de chaque participant. Les relations virtuelles sont donc un dépassement tout autant qu'une continuation. Elles sont totalement aléatoires. Elles sont hors temps et espace réels, mais 
le temps et l'espace réels sont toujours présents et on peut toujours y revenir à chaque instant.

70 2. La réalité virtuelle est un langage. C'est donc l'extension du langage humain. Une extension d'abord par un langage-machine, ou des langages-machine qui donnent à leurs possesseurs des pouvoirs énormes dans la machine, sur la machine, dans ce monde virtuel. Mais c'est aussi l'extension de la parole, de l'écriture, de l'expression, de l'échange et de la confrontation de mots, mais pas seulement de mots. Aussi de formes, d'objets, d'images, etc. C'est une forme d'expression humaine qui dépasse les limites de l'expression dans le monde réel.

71 3. Un univers de réalité virtuelle est l'extension du monde réel, de l'imagination des utilisateurs, des désirs, pulsions et fantasmes de ces mêmes utilisateurs. On peut déformer toutes les formes de toutes les façons paramétriquement imaginables, et on peut nier des règles physiologiques naturelles et créer des êtres fantastiques.

72 4. La réalité virtuelle est un média total. Elle est donc l'extension d'individus totaux, de sociétés totales et d'activités humaines totales. Chaque individu peut ainsi se créer une persona en continuité, en dépassement ou en contraste par rapport à sa personne réelle. Chaque site peut créer une société virtuelle en continuité, en dépassement ou en contraste par rapport à la réalité réelle extérieure. Chacun peut apporter son idéologie et ses savoirs réels, mais aussi changer d'idéologie et acquérir et utiliser des savoirs nouveaux qu'il peut ensuite réinvestir dans sa personne réelle, ou simplement laisser dans sa persona qui en devient ainsi le dépositaire. C'est d'ailleurs dans cette dimension que la réalité virtuelle jouera un jour un rôle capital dans le traitement des troubles, pathologiques ou non, du comportement humain.

\section{Conclusion}

Visual space is uniform, continuous, and connected. The rational man in our Western culture is a visual man. The fact that most conscious experience has little "visuality" in it is lost on him. Ours is a brand new world of allatonceness. "Time" has ceased, "space" has vanished. We now live in a global village... a simultaneous happening. (MacLuhan $1967: 63$ )

Que MacLuhan se rassure. Cela n'est pas vrai que du monde occidental. Les Japonais ont inventé le concept de Vizthink (visual thinking). L'humanité tout entière ne serait pas si elle n'avait pas développé sa vision et réalisé cette vision de trois façons : par des actions lui permettant de survivre, même dans des conditions impossibles ; par des représentations picturales du monde, et aucune civilisation n'est dépourvue d'images; et par des représentations conceptuelles du monde qui se réalisent dans le langage, outil fondamental pour la construction, la conservation et la transmission du savoir sans lequel l'humanité ne serait jamais sortie de l'animalité. Les langages scientifiques ont été des moyens de développer le savoir de l'homme. Les langages-machine le sont tout autant, mais en plus ils sont des moyens de développer et de dépasser la conscience de l'homme, son individualité, sa liberté et sa richesse personnelle.

Nous venons de poser trois niveaux de langage: le langage naturel qui conceptualise la vision du monde ; le langage scientifique qui construit le savoir sur le monde ; le langagemachine qui démultiplie la puissance visionnaire du premier en allant au-delà de la réalité, et la puissance explicative et découvrante du second par un traitement toujours plus rapide et fort des opérations d'analyse et de synthèse intellectuelles et scientifiques. 
Être timoré ou réservé face à cette révolution technologique qu'est la réalité virtuelle, quels que soient les problèmes qu'il faille affronter et résoudre, ne peut que laisser se développer ce média de façon sauvage. L'école à un rôle capital à jouer, suivant la procédure que nous avons donnée dans cet article, ou d'autres procédures à inventer, pour permettre un développement rapide et riche de l'utilisation du média comme un outil humanisant et non comme un jouet aliénant, car, comme toute technique, elle peut être positive ou négative dans ses utilisations et applications.

\section{BIBLIOGRAPHIE}

Connelly, Michael. 1996. The Poet. Londres : Orion.

Coulardeau, Jacques. 1994. « La Culture de la réalité virtuelle ». ASp 5-6, 111-120.

Coulardeau, Jacques. 1995. « Computing English: The converging metaphors ». ASp 7-10, 41-56.

Coulardeau, Jacques. 1998a « The four basic cognitive learning strategies ». In Actes du XVI

Congrès international des linguistes, édition en CD-ROM. Paris : Elsevier.

Coulardeau, Jacques. 1998b. « Stratégies cognitives et multimédia. Individualisation pédagogique dans le collectif classe ». In Actes de la $4^{e}$ Biennale de l'éducation et de la formation, édition en CDROM. Paris : INRP.

Foucault, Michel. 1979. Discipline and Punish. Trans. Sheridan, New York : Elsevier.

Holmes, David (dir.). 1997. Virtual Politics, Identity \& Community in Cyberspace. Londres : Sage Publications.

IBM Dictionary of Computing. 1994. New York : MacGraw-Hill (International ed.).

Jones, Steven G. (dir.). 1997. Virtual Culture, Identity and Communication in Cybersociety. Londres : Sage Publications.

Kay, Alan. 1992. « Mind Melding ». In Byte Outlook 92. New York : MacGraw-Hill.

King, Stephen. 1978. « The Lawnmower Man ». In Nightshift. New York : Doubleday,.

King, Stephen. 1980. Firestarter. New York : Viking.

King, Stephen. 1990. The Stand. New York : Doubleday.

King, Tabitha. 1993. One On One. New York : Signet.

MacLuhan, Marshall \& Quentin Fiore. 1967. The Medium is the Massage. An Inventory of Effects. New York : Bantam Books.

Rheingold, Howard. Virtual Reality, New York : Simon and Schuster, 1992.

Stanek, William. 1996. HTML, Java, CGI, VRML, SGML, Création de sites Web.Paris : Simon and Schuster/ Macmillan.

Straub, Peter. 1993. The Throat. New York : Signet.

Vygotski, Lev Sémionovitch. 1985. Pensée et Langage. Paris : Messidor. 


\section{RÉSUMÉS}

Nous avons découvert à Bournemouth (Grande-Bretagne) le rôle capital de la culture de l'apprenant comme motivation pour celui-ci, et dans cette culture, l'informatique tient une place centrale. La réalité virtuelle (RV) pour l'Internet se fonde sur deux langages-machine, le VRML et Java. Ce sont des langues industrielles pures, relayées par des langues pédagogiques en direction du grand public. La possession de ces langues ouvre un monde virtuel de masse qui représente une industrie et un commerce au développement exponentiel. Mais la pratique de la VR sur l'Internet rend largement caduques les approches de Vygotski (zone proche de développement) et de Lacan (l'Autre et l'Autorité voient leur position systémique dans le carré du sujet totalement déplacée). Nous franchissons une limite en pédagogie, fondée sur l'autocentrage et le giovannisme de l'apprenant: première étape d'une révolution didactique. Cela démultiplie le processus d'acquisition du savoir et tous les dangers envisageables ne sont que des résistances d'arrière-garde.

We discovered in Bournemouth, Great-Britain, the essential role of the learner's culture in his motivation, and the core of it is informatics. Virtual reality (VR) on the Internet is based on two machine languages, VRML and Java. They are pure industrial languages, relayed to the public by pedagogical discourses. Possessing them opens a mass virtual world nurturing exponentially growing industry and commerce. But the use of VR on the Internet invalidates Vygotski's zone of proximal development and breaks up Lacan's square of the subject by expelling the Other and his Authority out of it. We are introduced to a pedagogy of the self-centered and giovannistic learner: first stage of a didactic revolution. This amplifies the learning process, and all suggested dangers are nothing but anachronistic misinspired ideas.

\section{INDEX}

Mots-clés : auto-centré, auto-initié, auto-intéressé, auto-motivé, autorité, giovannisme, interaction, Internet, ludique, phallique, pulsionnel, réalité virtuelle, système-auteur, zone proche de développement

Keywords : authoring system, authority, giovannistic, impulsive, ludic, phallic, self-centered, self-initiated, self-interested, self-motivated, virtual reality, zone of proximal development

\section{AUTEUR}

\section{JACQUES COULARDEAU}

Jacques Coulardeau, docteur de l'université, a choisi pendant très longtemps de travailler dans le secondaire, en particulier technique industriel, et dans le premier cycle. Il a aussi officié en Langues étrangères appliquées (Université de Lille 3) et dans la formation continue (GRETA). Il participe, à titre divers, à de nombreuses associations linguistiques et culturelles, la plupart internationales, y compris l'Association internationale de psychomécanique, d'anglais de spécialité et de didactique des langues. Il est spécialiste de la littérature multimédia fantastique anglo-saxonne. dondaine@orange.fr 